\title{
Multi-phase powder patterns in characterization of new solid-state materials
}

\author{
$\underline{\text { Vladimir V. Chernyshev }}^{1}$, Vera I. Isaeva ${ }^{2}$, Anna A. Lobova ${ }^{1}$
}
${ }^{1}$ Department Of Chemistry, M.V. Lomonosov Moscow State University, Moscow, Russian Federation, ${ }^{2} \mathrm{~N}$. D. Zelinsky Institute of Organic Chemistry Russian Academy of Sciences, Moscow, Russian Federation
E-mail: vladimir@struct.chem.msu.ru

Structural characterization of any new material is an important step in understanding of its properties and their controllable tuning during the production on industrial scale. Unfortunately, quite often the early exploratory stages of the synthesis of new solid-state materials results in powder samples, which produce multi-phase X-ray patterns. What is the multi-phase powder pattern for us - temporary inconvenience or a path to new crystalline forms? Those who hold the first view (temporary inconvenience), trying to get a pure substance, improving the synthesis. Undoubtedly, this is the best way to structural characterization of the new crystalline form, although it may require a huge time costs. We adhere to the second point of view and use each multi-phase powder diffraction pattern for the search and selection of the peaks inherent to the new crystalline phase(s). Having a set of three or more multi-phase patterns, each of which contains diffraction peaks from the new crystalline phase, we are trying to establish its crystal structure. This approach has helped us in our work with $\mathrm{Zn}$ (II)-based MOFs with pyridine-2,5-dicarboxylate linkers, when the crystal structure and composition of the novel phases were established from the multi-phase synchrotron powder diffraction patterns [1]. Also, dealing with the cryomodified powder samples of dehydroepiandrosterone (DHEA), we were able to establish new crystal structures of anhydrous DHEA forms III and VII from multi-phase patterns measured on the laboratory powder diffractometer [2]. Recently, we have succeeded in the synthesis of single-phase sample of DHEA form III, and its powder pattern confirmed the correctness of the previously determined structure. Currently we are working with various samples of MOFs, processing their multi-phase powder patterns measured before and after their use in the catalysis. The obtained results will be presented and discussed.

This research was supported by the Russian Ministry of Science and Education, grant No. RFMEFI61616X0069.

[1] Isaeva, V.I. et al. (2013). Crystal Growth Des. 13, 5305-5315.

[2] Chernyshev, V.V. et al. (2016). Crystal Growth Des. 16, 1088-1095.

Keywords: metal-organic frameworks, structure determination from powder diffraction data 